\title{
Sensitivity and specificity of an indirect enzyme-linked immunoassay for the diagnosis of Brucella canis infection in dogs
}

\author{
N. E. LUCERO, G. I. ESCOBAR, S. M. AYALA and G. LOPEZ* \\ Brucellosis Laboratory, ANLIS Dr C. G. Malbrán, Avda Velez Sarsfield 563, 1281 Buenos Aires and*Zoonosis \\ Center, Municipalidad Lomas de Zamora, 12 de octubre 1060, Banfield, Argentina
}

\begin{abstract}
The diagnosis of $B$. canis infection in dogs is based on bacteriological examination and serological methods including agglutination and gel diffusion tests. Bacteriological studies are the only methods that have been considered specific but, as intermittent periods of abacteraemia may occur, a negative blood culture cannot be used as a criterion for excluding canine brucellosis. Close contact between people and infected dogs increases the risk of transmission; however, its impact on public health is probably underestimated due to lack of reporting and inadequate diagnostic services. This paper describes an indirect enzyme-linked immunoassay (IELISA) procedure for the diagnosis of brucellosis caused by $B$. canis in a population of normal and infected dogs previously screened by the buffered plate antigen test (BPAT) and rapid slide agglutination test (RSAT). The serological survey was performed with 446 field sera. The 270 sera from the asymptomatic group found negative by BPA, RSAT and blood culture showed IELISA specificities of $96.7 \%$ and $\mathbf{1 0 0 \%}$, respectively, when cut-off values of OD 0.237 and 0.281 were selected. For 52 sera from culture-positive dogs, IELISA sensitivity was $100 \%$ with cut-off values of $\mathrm{OD}_{414} \quad 0.237$ and 0.281 . $\mathrm{OD}_{414} 0.281$ was selected because this value provided the highest accuracy with minimal false-negative and false-positive results. This cut-off value was used to study 124 blood culture-negative but RSAT positive sera. IELISA produced 107 positive results; the 17 sera that were negative by IELISA presented a wide range of reactivities by RSAT ( 2 were RSAT positive at 1 in 2 dilution and 15 were weakly positive with pure serum). These samples were probably from animals at an early stage of the infection or were false-positive results. The IELISA described here detects IgG and IgA antibodies that are useful for evaluating the clinical status of dogs. Although RSAT is a practical screening test, a supplementary technique such as IELISA should be used on all positive RSAT samples to ensure diagnostic specificity. Furthermore, people in contact with infected dogs could be investigated for possible transmission. The procedure described in this study was relatively simple and could have widespread applications.
\end{abstract}

\section{Introduction}

Transmission of Brucella canis commonly occurs by contact with products of abortion or subsequent vaginal discharges. Infected males harbour organisms in the prostate gland and epididymides for many months after the bacteraemia has ceased and may disseminate the disease in semen when they breed [1]. Clinical signs are not adequate to diagnose canine brucellosis, as

Received 31 Jan. 2002; accepted 8 March 2002.

Corresponding author: Dr N. E. Lucero (e-mail: nidia@ elsitio.net). many infected males are clinically normal. However, the infection may be suspected when there is a history of abortions, diskospondylitis or poor reproductive performance in either sex.

Serological tests are the methods most commonly used to evaluate the status of dogs before breeding or whenever brucellosis is suspected. Blood cultures are essential for diagnosis, especially if serological results are ambiguous. The serological tests usually used are the rapid slide agglutination test (RSAT) and the tube agglutination test (TAT) (both with 2-mercaptoethanol). These tests are sensitive but many false-positive results 
have been found. Agar gel immunodiffusion (AGID) has been used but sometimes the precipitin lines are difficult to interpret. More recently, indirect enzymelinked immunoassay (IELISA) with cytoplasmic protein antigen, hot saline extracts or cell-wall antigens have been proposed [2-5].

Because of the threat of transmitting the disease to man, dogs suspected of having brucellosis should be investigated promptly. This paper describes an IELISA procedure for the diagnosis of brucellosis caused by $B$. canis in a population of normal and infected dogs previously screened by buffered plate antigen test (BPAT) and RSAT. The IELISA cut-off value was determined and the performance of the tests in terms of diagnostic specificity and sensitivity was analysed.

\section{Materials and methods}

\section{Serological tests}

The RSAT screening tests were performed as described previously [3], but with serial serum dilutions to find the final titre. Briefly, $10 \mu \mathrm{l}$ of serum dilution were mixed with $10 \mu \mathrm{l}$ of antigen on a $25 \times 75-\mathrm{mm}$ glass slide for 1-2 min and results were read with a $10 \times$ microscope objective, including a control standard serum whose titre was known. The antigen was prepared at ANLIS Dr C. G. Malbrán from the strain (M-) variant of B. canis kindly provided by Professor L. Carmichael who also supplied the antigen used as reference (L. Carmichael, personal communication). The buffered plate agglutination test (BPAT), Rose Bengal test (RB) and plate agglutination test (PAT) were done as described previously [6] with an antigen prepared at ANLIS Dr C. G. Malbrán from B. abortus strain 1119-3.

\section{IELISA}

Antigen. The antigen was obtained from the (M-) variant of $B$. canis by the procedure described previously for $B$. ovis $[7,8]$. Briefly, B. canis saline extract was prepared as described by Myers et al. [9], then centrifuged at $254000 \mathrm{~g}$ in a Kontron Instrument Ultra Centrifuge in a TFT 45.94 rotor for $4 \mathrm{~h}$ at $4^{\circ} \mathrm{C}$. The pellet was dissolved in $\mathrm{PBS}, \mathrm{pH} 7.2$, frozen at $-20^{\circ} \mathrm{C}$ and used at 1 in 2000 dilution after $\mathrm{OD}_{414}$ readings of various antigen dilutions with strongly positive, weakly positive and negative sera as controls. The control sera were from dogs with positive isolation of $B$. canis and positive RSAT with a titre of 1 in 128 and 1 in 8 , respectively. The negative serum was from a healthy dog negative in RSAT and BPAT.

Conjugate. A lyophilised horseradish peroxidaseconjugated protein $\mathrm{A} / \mathrm{G}$ was obtained from ImmunoPure (Pierce Lb) and used at 1 in 20000 after titration with strongly positive, weakly positive and negative dog sera.
Procedure. The antigen diluted in $0.06 \mathrm{M}$ sodium carbonate buffer ( $\mathrm{pH}$ 9.6) was passively coated on to polystyrene plates (Nunc 2-69620, Denmark) at $50 \mu \mathrm{l} /$ well and incubated for $18 \mathrm{~h}$ at room temperature and then washed five times in $0.01 \mathrm{M}$ phosphate-buffered saline (PBS) containing Tween $200.05 \%$, pH 7.2 $(\mathrm{PBS} / \mathrm{T})$. Control and test sera were added at 1 in 100 in $\mathrm{PBS} / \mathrm{T}, 50 \mu \mathrm{l} /$ well, for $1 \mathrm{~h}$ at room temperature. After five washes in $\mathrm{PBS} / \mathrm{T}$, appropriately diluted horseradish peroxidase-conjugated protein $\mathrm{A} / \mathrm{G}$ was added, $50 \mu \mathrm{l} / \mathrm{well}$, and incubated for $1 \mathrm{~h}$ at room temperature. After five washes in $\mathrm{PBS} / \mathrm{T}$, the final step was the addition of $100 \mu \mathrm{l}$ of chromogenic substrate

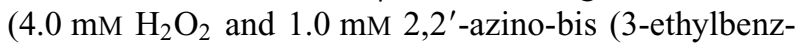
thiazoline-6-sulphonic acid) diammonium salt in $0.05 \mathrm{M}$ citrate buffer, $\mathrm{pH} 4.5$ ) per well. The plate was shaken continuously on an orbital shaker and, after $10 \mathrm{~min}$, the OD was measured at $414 \mathrm{~nm}$ in a photometer (Labsystems Multiskan EX microplate reader) with $100 \mu \mathrm{l}$ of chromogenic substrate in a plate as a control for the microplate reader. When the test is positive, colour develops.

\section{Bacteriological studies}

Brucella organisms were isolated as described previously [1] from a blood culture, semen or vaginal discharge. The isolates were typed as recommended by the International Committee on Systematic Bacteriology (ICBN) Subcomittee on Taxonomy of the Genus Brucella [10] at ANLIS Dr C. G. Malbrán.

\section{Canine sera}

The 446 sera included in the study were divided into the following groups. (i) Brucella-infected dogs: 52 sera were from dogs with confirmed brucellosis by clinical examination, serology and positive cultures. (ii) Asymptomatic dogs: 270 sera were from healthy dogs with no clinical or epidemiological evidence of brucellosis plus negative blood culture and negative serological tests such as RSAT and BPAT. (iii) Dogs with suspected brucellosis: 124 sera from dogs with clinical symptoms compatible with brucellosis, showing positive results to RSAT at any titre but negative blood culture.

\section{Data analysis}

Diagnostic specificity and sensitivity were determined initially with $95 \%$ confidence limits by plotting the data for negative and positive samples on a frequency histogram. The data were subsequently analysed by receiver-operator characteristics (ROC) analysis [11].

\section{Results}

The 270 sera negative in IELISA had a mean $\mathrm{OD}_{414}$ value of 0.148 (SD 0.043). Fig. 1 shows the frequency 


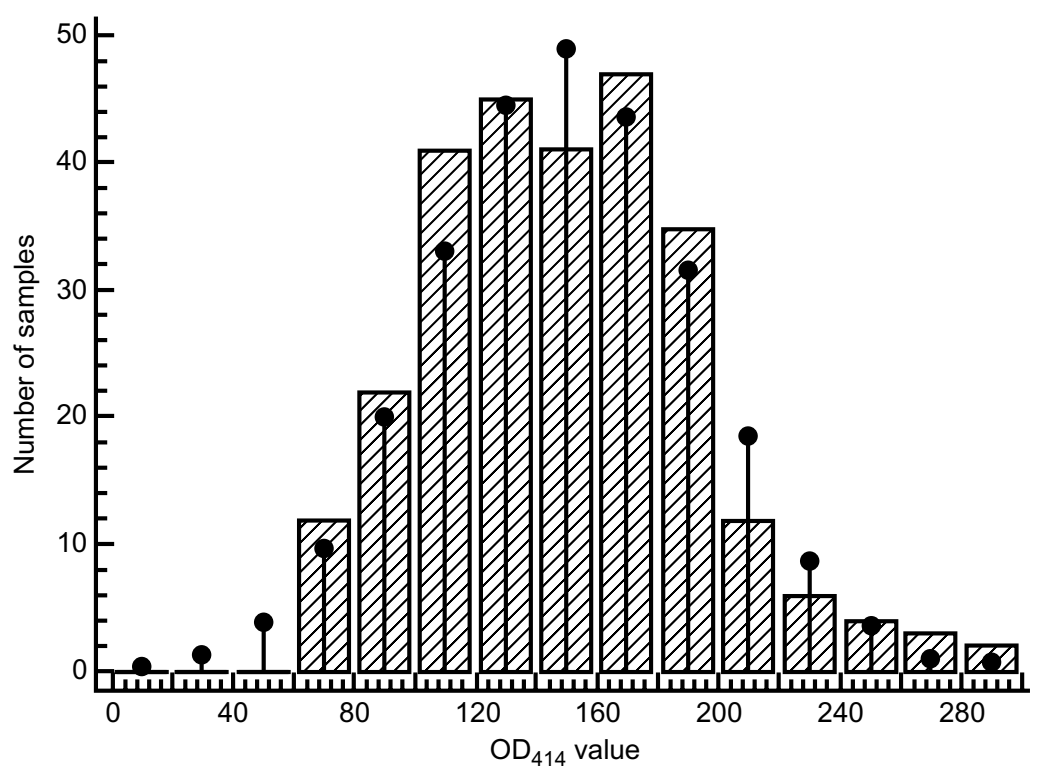

Fig. 1. Frequency distribution of IELISA results with 270 serum samples negative for antibody to $B$. canis.

distribution of these sera. Therefore, a cut-off value of $\mathrm{OD}_{414} \quad 0.236 \quad(2 \mathrm{SD})$ or $\mathrm{OD} \quad 0.279 \quad$ (3 $\mathrm{SD}$ ) was established and then confirmed with 52 positive sera from Brucella-infected dogs and 270 negative sera from asymptomatic dogs by ROC curve (Fig. 2). The $\mathrm{OD}_{414} 0.281$ cut-off value resulted in IELISA sensitivity and specificity of $100 \%$.

Table 1 shows the serological test results for the sera of 52 dogs with positive isolation of $B$. canis; most of the isolates were from blood culture except dog 46, which was positive by swab of vaginal discharges. Of the 124 sera from dogs suspected of having brucellosis, six

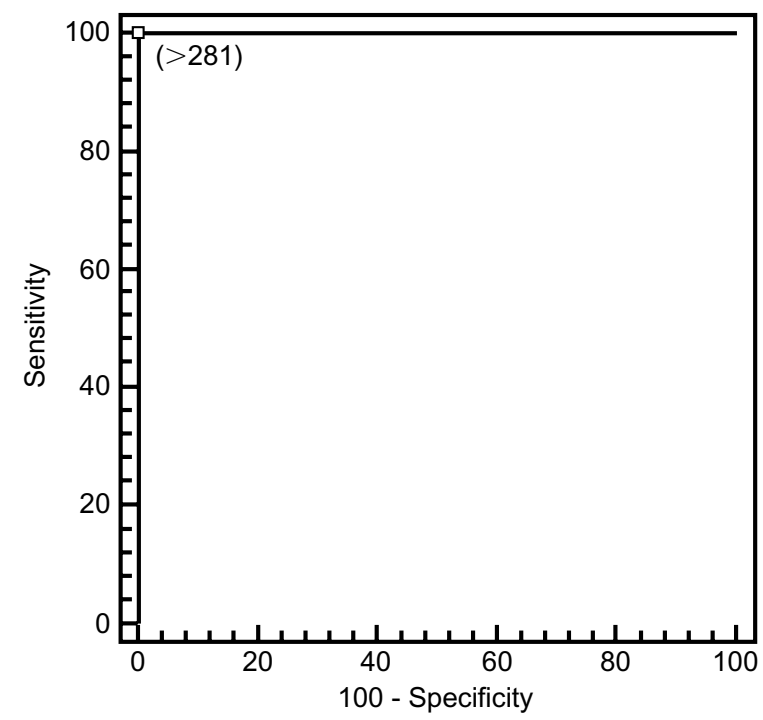

Fig. 2. ROC analysis plotting percent sensitivity (y axis) against 100-specificity (x axis) for various cut-off values. From the data a cut-off value of $\mathrm{OD}_{414} 0.281$ provides specificity and sensitivity values of $100 \%$. with weak or positive BPA titres were studied by PAT and RBT. Only one was weakly positive in RB and PAT at a dilution of 1 in 50. All six sera were positive in both IELISA and RSAT. Of 118 sera negative by BPA, 101 were positive by IELISA, showing low, moderate or high titres by RSAT. Of the 17 sera negative in IELISA, 2 were positive by RSAT at a dilution of 1 in 2 and 15 were weakly positive as pure serum.

\section{Discussion}

Canine brucellosis is an insidious disease that may be suspected when abortions occur in the last trimester or when epididymitis and testicular atrophy are observed in male dogs. These may be infertile and may show orchidism with varying degrees of prostatitis. However, many infected dogs are clinically normal. Close contact between man and infected dogs increases the risk of transmission; however, the impact on public health is probably underestimated because of lack of reporting and inadequate diagnostic services. The diagnosis of $B$. canis infection in dogs is based on bacteriological examination and serological methods, usually agglutination and gel diffusion tests. Bacteriological studies are the only method that has been considered specific but, as intermittent periods of abacteraemia may occur, a negative blood culture cannot be used as a criterion for excluding canine brucellosis. Agglutination tests have good sensitivity but their lack of specificity and the occurrence of false positive serological results make a specific test necessary.

The objectives of this study were to ascertain the usefulness of IELISA for the diagnosis of brucellosis caused by $B$. canis and to determine the cut-off value. The serological survey was performed with 446 field 
Table 1. Serological response of sera from B. canis culture-positive $\operatorname{dog} \mathrm{s}^{*}$ to IELISA and RSAT

\begin{tabular}{|c|c|c|c|}
\hline Serum no. & $\mathrm{RSAT}^{\dagger}$ & $\begin{array}{l}\text { IELISA } \\
\left(\mathrm{OD}_{414}\right)\end{array}$ & Isolated from \\
\hline 1 & 128 & 0.930 & Blood \\
\hline 2 & 8 & 0.749 & Blood \\
\hline 3 & 8 & 0.762 & Blood \\
\hline 4 & 16 & 0.687 & Semen and blood \\
\hline 5 & 4 & 0.531 & Blood \\
\hline 6 & 64 & 0.977 & Blood \\
\hline 7 & 32 & 0.691 & Blood \\
\hline 8 & 8 & 0.938 & Blood \\
\hline 9 & 32 & 0.673 & Blood \\
\hline 10 & 64 & 0.751 & Blood \\
\hline 11 & 32 & 0.902 & Blood \\
\hline 12 & 8 & 0.961 & Blood \\
\hline 13 & 8 & 0.982 & Semen and blood \\
\hline 14 & 4 & 0.898 & Blood \\
\hline 15 & 2 & 0.503 & Blood \\
\hline 16 & 4 & 0.870 & Blood \\
\hline 17 & 8 & 0.548 & Blood \\
\hline 18 & 2 & 0.567 & Blood \\
\hline 19 & 2 & 0.562 & Blood \\
\hline 20 & 8 & 0.582 & Blood \\
\hline 21 & 32 & 1.070 & Blood \\
\hline 22 & 4 & 0.494 & Blood \\
\hline 23 & 2 & 0.405 & Blood \\
\hline 24 & 32 & 0.838 & Blood \\
\hline 25 & 16 & 0.653 & Blood \\
\hline 26 & 4 & 0.706 & Blood \\
\hline 27 & 4 & 0.729 & Blood \\
\hline 28 & 8 & 0.746 & Blood \\
\hline 29 & 2 & 0.502 & Blood \\
\hline 30 & 4 & 0.615 & Blood \\
\hline 31 & 64 & 0.793 & Blood \\
\hline 32 & 4 & 0.356 & Blood \\
\hline 33 & 32 & 1.180 & Blood \\
\hline 34 & 4 & 0.950 & Blood \\
\hline 35 & 2 & 0.668 & Blood \\
\hline 36 & 32 & 0.744 & Blood \\
\hline 37 & 32 & 0.654 & Blood \\
\hline 38 & 16 & 0.456 & Blood \\
\hline 39 & 8 & 0.582 & Blood \\
\hline 40 & 32 & 0.430 & Blood \\
\hline 41 & 256 & 0.579 & Blood \\
\hline 42 & 32 & 0.506 & Blood \\
\hline 43 & 64 & 0.491 & Blood \\
\hline 44 & 32 & 0.412 & Blood \\
\hline 45 & 256 & 0.498 & Blood \\
\hline 46 & 128 & 0.626 & Vaginal swab \\
\hline 47 & 8 & 0.432 & Vaginal swab and blood \\
\hline 48 & 32 & 0.574 & Vaginal swab and blood \\
\hline 49 & 16 & 0.590 & Blood \\
\hline 50 & 64 & 0.640 & Blood \\
\hline 51 & 32 & 0.946 & Blood \\
\hline 52 & 16 & 0.489 & Blood \\
\hline
\end{tabular}

*All sera were negative for BPAT.

${ }^{\dagger}$ Serum dilutions.

sera. The 270 sera from the asymptomatic group found negative by BPA, RSAT and blood culture showed IELISA specifications of $96.7 \%$ and $100 \%$, respectively, when cut-off values of $\mathrm{OD}_{414} 0.237$ and 0.281 were selected. For 52 sera from culture-positive dogs, the IELISA sensitivity was $100 \%$ with cut-off values of $\mathrm{OD}_{414} 0.237$ and 0.281 .

The cut-off value of $\mathrm{OD}_{414} 0.281$ was selected because this value has the highest accuracy plus minimal false- negative and false-positive results. With this cut-off value, 124 blood-culture negative but RSAT-positive sera were studied and IELISA produced 107 positive results. The 17 sera that were negative by IELISA presented a wide range of reactivities by RSAT ( 2 were RSAT positive at a dilution of 1 in 2 and 15 were weakly positive with pure serum); these samples were probably from animals at an early stage of the infection or were false-positive results. Of six sera that were positive by both BPA and RSAT, only one was weakly positive in RB, but all six sera were strongly positive in RSAT and IELISA. Some overlapping in the detection of antibodies against rough and smooth Brucella strains has been reported [4], but in the present study this phenomenon was observed in only $6(1.34 \%)$ of 446 cases.

The IELISA described here detects $\operatorname{IgG}$ and $\operatorname{IgA}$ antibodies that are useful for evaluating the clinical status of dogs. Although the RSAT is a practical screening test, a supplementary technique such as IELISA should be performed on all positive RSAT samples to ensure diagnostic specificity. Furthermore, it is suggested that studies of people in contact with infected dogs should be performed to investigate possible transmission.

The data presented clearly indicate that IELISA was more sensitive and specific than RSAT. The procedure described in this study was relatively simple to perform and may have widespread applications.

We are very grateful to Dr Klaus Nielsen from the Canadian Food Inspection Agency, Animal Research Institute, Ontario, Canada, for critically reading the manuscript.

\section{References}

1. Carmichael LE, Shin SJ. Canine brucellosis: a diagnostician's dilemma. Semin Vet Med Surg (Small Anim) 1996; 11: $161-165$.

2. Baldi PC, Wanke MM, Loza ME, Monachesi N, Fossati CA. Diagnosis of canine brucellosis by detection of serum antibodies against an $18 \mathrm{kDa}$ cytoplasmic protein of Brucella spp. Vet Microbiol 1997; 51: 273-281.

3. Carmichael LE, Joubert JC. A rapid slide agglutination test for the serodiagnosis of Brucella canis infection that employs a variant (M-) organism as antigen. Cornell Vet 1987; 77: 3-12.

4. Mateau-de-Antonio EM, Martin M, Soler M. Use of indirect enzyme-linked immunosorbent assay with hot saline solution extracts of a variant (M-) strain of Brucella canis for diagnosis of brucellosis in dogs. Am J Vet Res 1993; 54: 1043-1046.

5. Serikawa T, Iwaki S, Mori M, Muraguchi T, Yamada J. Purification of a Brucella canis cell wall antigen by using immunosorbent columns and use of the antigen in enzymelinked immunosorbent assay for specific diagnosis of canine brucellosis. J Clin Microbiol 1989; 27: 837-842.

6. Lucero NE, Bolpe JE. Buffered plate antigen test as a screening test for diagnosis of human brucellosis. $J$ Clin Microbiol 1998; 36: 1425-1427.

7. Suarez CE, Pacheco GA, Vigliocco AM. Characterization of Brucella ovis surface antigens. Vet Microbiol 1988; 18: 349-356.

8. Vigliocco AM, Silva Paulo PS, Mestre J et al. Development and validation of an indirect enzyme immunoassay for detection of ovine antibody to Brucella ovis. Vet Microbiol 
1997; 54: 357-368.

9. Myers DM, Jones LM, Varela Diaz VM. Studies of antigens for complement fixation and gel diffusion tests in the diagnosis of infections caused by Brucella ovis and other Brucella. Appl Microbiol 1972; 23: 894-902.

10. Corbel MJ, Brinley-Morgan WJ. Genus Brucella, Meyer and
Shaw 1920, 173 AL, In: Krieg NR, Holt JG (eds) Bergey's manual of systematic bacteriology, vol 1. Baltimore, MD, Williams \& Wilkins 1984: 377-388.

11. Schoonjans F, Zalata A, Depuydt CE, Comhaire FH. MedCalc: a new computer program for medical statistics. Comput Methods Programs Biomed 1995; 48: 257-262. 